\title{
On the Zeros of Dirichlet $L$-Functions II
}

by

\section{Akio FuJII}

\author{
(Received September 18, 2003)
}

\section{§1. Introduction}

Here we are concerned with the distribution of the zeros of Dirichlet $L$-functions. Corresponding to $\zeta$-function theorems proved in our previous work [3], analogous theorems will be presented for $L$-functions when we consider their conductors as a variable.

Let $L(s, \chi)$ be the Dirichlet $L$-function attached to a primitive Dirichlet character $\chi$ $\bmod q(>1)$. We denote the non-trivial zeros of $L(s, \chi)$ by $\rho(\chi)=\beta(\chi)+i \gamma(\chi)$ with real numbers $\beta(\chi)$ and $\gamma(\chi)$. When $T \neq \gamma(\chi)$ for any $\gamma(\chi)$, we put

$$
S(T, \chi)=\frac{1}{\pi} \arg L\left(\frac{1}{2}+i T, \chi\right)
$$

where the argument is obtained by the continuous variation along the straight line $s=$ $\sigma+i T$, when $\sigma$ varies from $+\infty$ to $\frac{1}{2}$, starting with the value zero. When $T=\gamma(\chi)$, then we put

$$
S(T, \chi)=\frac{1}{2}(S(T+0, \chi)+S(T-0, \chi))
$$

It is known that

$$
S(T, \chi)=O(\log q(1+T))
$$

and that under the Generalized Riemann Hypothesis (cf. Theorem 6 on p. 330 of Selberg [5])

$$
S(T, \chi)=O\left(\frac{\log q(1+T)}{\log \log q(1+T)}\right) .
$$

In Fujii [2], we have been concerned with the average behavior of $S(T, \chi)$ and defined

$$
\tilde{S}_{m}(T, \chi)
$$

for $m \geq 0$ as follows. When $T \neq \gamma(\chi)$, then we put

$$
\tilde{S}_{0}(T, \chi)=S(T, \chi)
$$

and

$$
\tilde{S}_{m}(T, \chi)=\int_{0}^{T} \tilde{S}_{m-1}(t, \chi) d t+C_{m}(\chi)
$$

for any integer $m \geq 1$, where $C_{m}(\chi)$ 's are the constants defined by 


$$
C_{2 k-1}(\chi)=(-1)^{k-1} \frac{1}{\pi} \underbrace{\int_{\frac{1}{2}}^{\infty} \int_{\sigma}^{\infty} \cdots \int_{\sigma}^{\infty}}_{2 k-1} \log |L(\sigma, \chi)|(d \sigma)^{2 k-1}
$$

for $m=2 k-1$ and

$$
C_{2 k}(\chi)=(-1)^{k} \underbrace{\int_{\frac{1}{2}}^{\infty} \int_{\sigma}^{\infty} \cdots \int_{\sigma}^{\infty}}_{2 k} \Im \log L(\sigma, \chi)(d \sigma)^{2 k}
$$

for $m=2 k$, respectively. When $T=\gamma(\chi)$, then we put

$$
\tilde{S}_{m}(T, \chi)=\frac{1}{2}\left(\tilde{S}_{m}(T+0, \chi)+\tilde{S}_{m}(T-0, \chi)\right) .
$$

We shall first describe the relation between $\tilde{S}_{m}(T, \chi)$ and the integral $I_{m}(T, \chi)$ which will be defined as follows. When $T \neq \gamma(\chi)$, then we put for $k \geq 1$

$$
I_{2 k-1}(T, \chi)=\frac{1}{\pi}(-1)^{k-1} \Re\{\underbrace{\int_{\frac{1}{2}}^{\infty} \int_{\sigma}^{\infty} \cdots \int_{\sigma}^{\infty}}_{2 k-1} \log L(\sigma+i T, \chi)(d \sigma)^{2 k-1}\}
$$

and

$$
I_{2 k}(T, \chi)=\frac{1}{\pi}(-1)^{k} \Im\{\underbrace{\int_{\frac{1}{2}}^{\infty} \int_{\sigma}^{\infty} \cdots \int_{\sigma}^{\infty}}_{2 k} \log L(\sigma+i T, \chi)(d \sigma)^{2 k}\} .
$$

When $T=\gamma(\chi)$, then we put for $m \geq 1$

$$
I_{m}(T, \chi)=\frac{1}{2}\left(I_{m}(T+0, \chi)+I_{m}(T-0, \chi)\right) .
$$

For $\sigma \geq \frac{1}{2}$ and $T>T_{o}$, let $N(\sigma, T, \chi)$ be the number of the zeros $\beta(\chi)+i \gamma(\chi)$ of $L(s, \chi)$ such that $\beta(\chi)>\sigma$ and $0 \leq \gamma(\chi)<T$ when $T \neq \gamma(\chi)$, the zeros with $\gamma(\chi)=0$ counting one half only. When $T=\gamma(\chi)$, then we put

$$
N(\sigma, T, \chi)=\frac{1}{2}((N(\sigma, T+0, \chi)+N(\sigma, T-0, \chi)) .
$$

In Fujii [2], we have shown the following results. We suppose in (I) and (II) below that $T>0$ and $\chi$ is a primitive Dirichlet character $\bmod q>1$.

I. We have

$$
\tilde{S}_{1}(T, \chi)=I_{1}(T, \chi)
$$

and for any integer $m \geq 2$

$$
\tilde{S}_{m}(T, \chi)=I_{m}(T, \chi)+2 \sum_{\substack{h+2 r=m \\ r \geq 1, h \geq 0}}(-1)^{r-1} \tilde{N}_{h, 2 r}(T, \chi),
$$

where we put for $h \geq 1$ and $r \geq 1$ 


$$
\tilde{N}_{h, 2 r}(T)=\underbrace{\int_{0}^{T} \cdots \int_{0}^{t}}_{h} \underbrace{\int_{\frac{1}{2}}^{1} \int_{\sigma}^{1} \cdots \int_{\sigma}^{1}}_{2 r} N(\sigma, t, \chi)(d \sigma)^{2 r}(d t)^{h}
$$

and for $h=0$ and $r \geq 1$

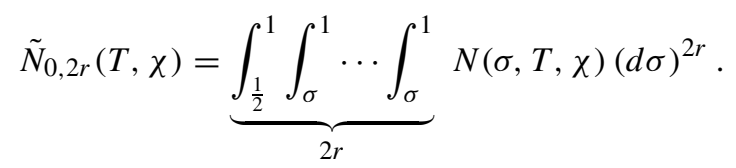

II-(i). We have for any integer $m \geq 1$,

$$
I_{m}(T, \chi) \ll \log q(1+|T|),
$$

where the constant involved in the upper bound may depend on $\mathrm{m}$.

II-(ii). We have

$$
\tilde{S}_{1}(T, \chi)=O(\log q(1+T))
$$

and for any integer $m \geq 2$,

$$
\tilde{S}_{m}(T, \chi)=2 \sum_{\substack{h+2 r=m \\ r \geq 1, h \geq 0}}(-1)^{r-1} \tilde{N}_{h, 2 r}(T, \chi)+O(\log q(1+T)) .
$$

II-(iii). Suppose further that $q \ll T^{\frac{1}{4}-\varepsilon}$ with an arbitrarily small positive $\varepsilon$. Then we have for any integer $m \geq 2$

$$
\tilde{S}_{m}(T, \chi) \ll \frac{T^{m-1}}{\log T} .
$$

II-(iv). (On the Generalized Riemann Hypothesis) We have for any integer $m \geq 1$,

$$
\tilde{S}_{m}(T, \chi)=O\left(\frac{\log q(1+T)}{(\log \log q(1+T))^{m+1}}\right) .
$$

II-(v). The following statement is equivalent to the Generalized Riemann Hypothesis for $L(s, \chi)$. For any integer $m \geq 3$, we have

$$
\tilde{S}_{m}(T, \chi)=o\left(T^{m-2}\right) \quad(T \rightarrow \infty) .
$$

We now turn our attensions to $q$-analogues. When we consider $q$-analogue in this article, we always assume, for simplicity as in pp. 281-340 of Selberg [5], that $q$ runs over the odd prime numbers. Selberg (cf. Theorem 8 on p. 336 of [5]) showed that for $|T| \leq q^{\frac{1}{4}-\varepsilon}, \varepsilon$ being a fixed arbitrarily small positive number $\leq \frac{1}{4}$,

$$
\sum_{\chi \neq \chi_{0}} S(T, \chi)=O(q)
$$


where $\chi$ runs over the Dirichlet characters $\bmod q$ and $\chi_{0}$ is the principal character $\bmod q$. Moreover, Selberg showed that for $|T| \leq q^{\frac{1}{4}-\varepsilon}$

$$
\sum_{\chi \neq \chi_{0}}(S(T, \chi))^{2 k}=\frac{(2 k) !}{(2 \pi)^{2 k} k !} q(\log \log q)^{k}+O\left(q(\log \log q)^{k-\frac{1}{2}}\right) .
$$

It has been our problem to extend these to $\tilde{S}_{m}(T, \chi)$ for $m \geq 1$. Concerning $\sum_{\chi \neq \chi_{0}} \tilde{S}_{m}(T, \chi)$, we [2] have shown the following results.

III-(i). For any integer $m \geq 1$ and for $|T| \leq q^{\frac{1}{4}-\varepsilon}$, we have

$$
\sum_{\chi \neq \chi_{0}} I_{m}(T, \chi)=O(q) \text {. }
$$

III-(ii). Suppose that $0<T \leq q^{\frac{1}{4}-\varepsilon}$ with an arbitrarily small positive number $\varepsilon \leq \frac{1}{4}$. Then we have

$$
\sum_{\chi \neq \chi_{0}} \tilde{S}_{1}(T, \chi)=O(q)
$$

For any integer $m \geq 2$, we have

$$
\begin{aligned}
\sum_{\chi \neq \chi_{0}} \tilde{S}_{m}(T, \chi) & =2 \sum_{\substack{h+2 r=m \\
r \geq 1, h \geq 0}}(-1)^{r-1} \sum_{\chi \neq \chi_{0}} \tilde{N}_{h, 2 r}(T, \chi)+O(q) \\
& =O\left(\frac{q T^{m-1}}{\log q}+q\right)
\end{aligned}
$$

III-(iii). For any integer $m \geq 2$ and for $0<T \ll(\log q)^{\frac{1}{m-1}}$, we have

$$
\sum_{\chi \neq \chi_{0}} \tilde{S}_{m}(T, \chi)=O(q) .
$$

III-(iv). (On the Generalized Riemann Hypothesis) For any integer $m \geq 0$ and for $0<T \ll e^{A(\log q)^{m+1}}$ with any positive constant $A$, we have

$$
\sum_{\chi \neq \chi_{0}} \tilde{S}_{m}(T, \chi)=O(q) .
$$

Here we are concerned with the mean value theorems on high powers of $\tilde{S}_{m}(t, \chi)$ and shall prove the following theorem which is a $q$-analogue of Theorem 2 in our previous work [3].

THEOREM. Suppose that $|T| \leq q^{\frac{1}{4}-\varepsilon}$, where $\varepsilon$ is any arbitrarily small positive number $\leq \frac{1}{4}$. Then for any integer $m \geq 1$ and $k \geq 1$, we have 


$$
\begin{aligned}
\sum_{\chi \neq \chi_{0}}\left(\tilde{S}_{m}(T, \chi)-2 \sum_{\substack{h+2 r=m \\
r \geq 1, h \geq 0}}(-1)^{r-1} \tilde{N}_{h, 2 r}(T, \chi)\right)^{2 k} \\
=\sum_{\chi \neq \chi_{0}}\left(I_{m}(T, \chi)\right)^{2 k} \\
=\frac{q}{(2 \pi)^{2 k}} \sum_{j=0}^{2 k}\left(\begin{array}{c}
2 k \\
j
\end{array}\right)(-1)^{(j+k)(m+1)} \\
\sum_{\substack{n_{l}=2 \\
n_{1} \cdots n_{j}=n_{j+1} \cdots n_{2 k}}}^{\infty} \frac{\Lambda\left(n_{1}\right) \cdots \Lambda\left(n_{2 k}\right)}{n_{1} \cdots n_{j}\left(\log n_{1} \cdots \log n_{2 k}\right)^{m+1}}+O\left(\frac{q}{\log q} R_{m}(q)\right),
\end{aligned}
$$

where $\Lambda(n)$ is the von-Mangoldt function defined by

$$
\Lambda(n)= \begin{cases}\log p & \text { if } n=p^{k} \text { with a prime } p \text { and an integer } k \geq 1 \\ 0 & \text { otherwise }\end{cases}
$$

and we put

$$
R_{m}(q)= \begin{cases}\sqrt{\log \log q} & \text { if } m=1 \\ 1 & \text { if } m \geq 2 .\end{cases}
$$

We may notice some direct consequences of this theorem.

COROLLARY 1. Suppose that $|T| \leq q^{\frac{1}{4}-\varepsilon}$, where $\varepsilon$ is any arbitrarily small positive number $\leq \frac{1}{4}$. Then for any integer $k \geq 1$, we have

$$
\begin{aligned}
& \sum_{\chi \neq \chi_{0}}\left(\tilde{S}_{1}(T, \chi)\right)^{2 k}=\frac{q}{(2 \pi)^{2 k}} \sum_{j=0}^{2 k}\left(\begin{array}{c}
2 k \\
j
\end{array}\right) \\
& \cdot \sum_{\substack{n_{l}=2 \\
n_{1} \cdots n_{j}=n_{j+1} \cdots n_{2 k}}}^{\infty} \frac{\Lambda\left(n_{1}\right) \cdots \Lambda\left(n_{2 k}\right)}{n_{1} \cdots n_{j}\left(\log n_{1} \cdots \log n_{2 k}\right)^{2}}+O\left(\frac{q \sqrt{\log \log q}}{\log q}\right) .
\end{aligned}
$$

COROLlary 2. (On the Generalized Riemann Hypothesis) Suppose that $m$ is an integer $\geq 2$ and $|T| \leq e^{A(\log q)^{m}}$, where $A$ is some positive absolute constant. Then for any integer $k \geq 1$, we have

$$
\begin{aligned}
\sum_{\chi \neq \chi_{0}}\left(\tilde{S}_{m}(T, \chi)\right)^{2 k}= & \frac{q}{(2 \pi)^{2 k}} \sum_{j=0}^{2 k}\left(\begin{array}{c}
2 k \\
j
\end{array}\right)(-1)^{(j+k)(m+1)} \\
& \cdot \sum_{\substack{n_{l}=2 \\
n_{1} \cdots n_{j}=n_{j+1} \cdots n_{2 k}}}^{\infty} \frac{\Lambda\left(n_{1}\right) \cdots \Lambda\left(n_{2 k}\right)}{n_{1} \cdots n_{j}\left(\log n_{1} \cdots \log n_{2 k}\right)^{m+1}}+O\left(\frac{q}{\log q}\right) .
\end{aligned}
$$




\section{§. Proof of Theorem.}

In the rest of this article, we shall prove Theorem. Hereafter we suppose that $|t| \leq$ $q^{\frac{1}{4}-\varepsilon}$, where $\varepsilon$ is any arbitrarily small positive number $\leq \frac{1}{4}$. We put

$$
X=q^{\frac{\varepsilon C}{k}}
$$

with some positive absolute constant $C$. We start with the following lemma which is proved in Fujii [2].

LEMMA. For any integer $m \geq 1$, we have

$$
\begin{aligned}
I_{m}(t, \chi)= & \frac{1}{\pi} \Im\left\{i^{m} \sum_{n<X^{3}} \frac{\Lambda_{X}(n) \chi(n)}{n^{\sigma_{\chi}+i t}(\log n)^{m+1}} \sum_{\nu=0}^{m} \frac{\left(\left(\sigma_{\chi}-\frac{1}{2}\right) \log n\right)^{\nu}}{\nu !}\right\} \\
& +O\left(\left(\sigma_{\chi}-\frac{1}{2}\right)^{m+1}\left|\sum_{n<X^{3}} \frac{\Lambda_{X}(n) \chi(n)}{n^{\sigma_{\chi}+i t}}\right|\right)+O\left(\left(\sigma_{\chi}-\frac{1}{2}\right)^{m+1} \log q(1+|t|)\right),
\end{aligned}
$$

where $\Lambda_{X}(n)$ is defined by

$$
\Lambda_{X}(n)= \begin{cases}\Lambda(n) & \text { for } 1 \leq n \leq X \\ \Lambda(n) \frac{\log ^{2} \frac{X^{3}}{n}-2 \log ^{2} \frac{X^{2}}{n}}{2 \log ^{2} X} & \text { for } X \leq n \leq X^{2} \\ \Lambda(n) \frac{\log ^{2} \frac{X^{3}}{n}}{2 \log ^{2} X} & \text { for } X^{2} \leq n \leq X^{3}\end{cases}
$$

and we put, as in pp. 323-325 of Selberg [5],

$$
\sigma_{\chi}=\frac{1}{2}+2 \max _{\rho(\chi)}\left(\beta(\chi)-\frac{1}{2}, \frac{2}{\log X}\right),
$$

$\rho(\chi)$ running over all zeros $\beta(\chi)+i \gamma(\chi)$ of $L(s, \chi)$ for which

$$
|t-\gamma(\chi)| \leq \frac{X^{3\left|\beta(\chi)-\frac{1}{2}\right|}}{\log X} .
$$

For convenience, we shall decompose the right hand side further as follows.

$$
\begin{aligned}
I_{m}(t, \chi)= & \frac{1}{\pi} \Im\left\{i^{m} \sum_{n<X} \frac{\Lambda(n) \chi(n)}{\sqrt{n} n^{i t}(\log n)^{m+1}}\right\} \\
& +\frac{1}{\pi} \Im\left\{i^{m} \sum_{X \leq n<X^{3}} \frac{\Lambda_{X}(n) \chi(n)}{\sqrt{n} n^{i t}(\log n)^{m+1}}\right\}
\end{aligned}
$$




$$
\begin{aligned}
& +\frac{1}{\pi} \Im\left\{i^{m} \sum_{n<X^{3}} \frac{\Lambda_{X}(n) \chi(n)}{n^{i t}(\log n)^{m+1}}\left(\frac{1}{n^{\sigma_{\chi}}}-\frac{1}{\sqrt{n}}\right)\right\} \\
& +\frac{1}{\pi} \Im\left\{i^{m} \sum_{\nu=1}^{m} \frac{\left(\sigma_{\chi}-\frac{1}{2}\right)^{\nu}}{\nu !} \sum_{n<X^{3}} \frac{\Lambda_{X}(n) \chi(n)}{n^{\sigma_{\chi}+i t}(\log n)^{m+1-\nu}}\right\} \\
& +O\left(\left(\sigma_{\chi}-\frac{1}{2}\right)^{m+1}\left|\sum_{n<X^{3}} \frac{\Lambda_{X}(n) \chi(n)}{n^{\sigma_{\chi}+i t}}\right|\right) \\
& +O\left(\left(\sigma_{\chi}-\frac{1}{2}\right)^{m+1} \log q(1+|t|) .\right.
\end{aligned}
$$

The last three sums are rewritten in the following form as in p. 334 of Selberg [5].

$$
\begin{aligned}
& \left|\frac{1}{\pi} \Im\left\{i^{m} \sum_{n<X^{3}} \frac{\Lambda_{X}(n) \chi(n)}{n^{i t}(\log n)^{m+1}}\left(\frac{1}{n^{\sigma_{\chi}}}-\frac{1}{\sqrt{n}}\right)\right\}\right| \\
& \quad=\left|\frac{1}{\pi} \Im\left\{i^{m} \int_{\frac{1}{2}}^{\sigma_{\chi}} \sum_{n<X^{3}} \frac{\Lambda_{X}(n) \chi(n)}{n^{\sigma^{\prime}+i t}(\log n)^{m}} d \sigma^{\prime}\right\}\right| \\
& \quad \ll\left(\sigma_{\chi}-\frac{1}{2}\right) X^{\sigma_{\chi}-\frac{1}{2}} \int_{\frac{1}{2}}^{\infty} X^{\frac{1}{2}-\sigma}\left|\sum_{n<X^{3}} \frac{\Lambda_{X}(n) \chi(n) \log (X n)}{n^{\sigma+i t}(\log n)^{m}}\right| d \sigma \\
& \left|\frac{1}{\pi} \Im\left\{i^{m} \sum_{\nu=1}^{m} \frac{\left(\sigma_{\chi}-\frac{1}{2}\right)^{\nu}}{\nu !} \sum_{n<X^{3}} \frac{\Lambda_{X}(n) \chi(n)}{n^{\sigma_{\chi}+i t}(\log n)^{m+1-\nu}}\right\}\right| \\
& \quad \ll \sum_{\nu=1}^{m}\left(\sigma_{\chi}-\frac{1}{2}\right)^{\nu} X^{\sigma_{\chi}-\frac{1}{2}} \int_{\frac{1}{2}}^{\infty} X^{\frac{1}{2}-\sigma}\left|\sum_{n<X^{3}} \frac{\Lambda_{X}(n) \chi(n) \log (X n)}{n^{\sigma+i t}(\log n)^{m+1-\nu}}\right| d \sigma
\end{aligned}
$$

and

$$
\begin{aligned}
& \left(\sigma_{\chi}-\frac{1}{2}\right)^{m+1}\left|\sum_{n<X^{3}} \frac{\Lambda_{X}(n) \chi(n)}{n^{\sigma_{\chi}+i t}}\right| \\
& \quad \ll\left(\sigma_{\chi}-\frac{1}{2}\right)^{m+1} X^{\sigma_{\chi}-\frac{1}{2}} \int_{\frac{1}{2}}^{\infty} X^{\frac{1}{2}-\sigma}\left|\sum_{n<X^{3}} \frac{\Lambda_{X}(n) \chi(n) \log (X n)}{n^{\sigma+i t}}\right| d \sigma .
\end{aligned}
$$

Thus we have

$$
\begin{aligned}
I_{m}(t, \chi)= & \frac{1}{\pi} \Im\left\{i^{m} \sum_{n<X} \frac{\Lambda(n) \chi(n)}{\sqrt{n} n^{i t}(\log n)^{m+1}}\right\} \\
& +\frac{1}{\pi} \Im\left\{i^{m} \sum_{X \leq n<X^{3}} \frac{\Lambda_{X}(n) \chi(n)}{\sqrt{n} n^{i t}(\log n)^{m+1}}\right\}
\end{aligned}
$$




$$
\begin{aligned}
& +O\left(\left(\sigma_{\chi}-\frac{1}{2}\right) X^{\sigma_{\chi}-\frac{1}{2}} \int_{\frac{1}{2}}^{\infty} X^{\frac{1}{2}-\sigma}\left|\sum_{n<X^{3}} \frac{\Lambda_{X}(n) \chi(n) \log (X n)}{n^{\sigma+i t}(\log n)^{m}}\right| d \sigma\right) \\
& +O\left(\sum_{\nu=1}^{m}\left(\sigma_{\chi}-\frac{1}{2}\right)^{\nu} X^{\sigma_{\chi}-\frac{1}{2}} \int_{\frac{1}{2}}^{\infty} X^{\frac{1}{2}-\sigma}\left|\sum_{n<X^{3}} \frac{\Lambda_{X}(n) \chi(n) \log (X n)}{n^{\sigma+i t}(\log n)^{m+1-\nu}}\right| d \sigma\right) \\
& +O\left(\left(\sigma_{\chi}-\frac{1}{2}\right)^{m+1} X^{\sigma_{\chi}-\frac{1}{2}} \int_{\frac{1}{2}}^{\infty} X^{\frac{1}{2}-\sigma}\left|\sum_{n<X^{3}} \frac{\Lambda_{X}(n) \chi(n) \log (X n)}{n^{\sigma+i t}}\right| d \sigma\right) \\
& +O\left(\left(\sigma_{\chi}-\frac{1}{2}\right)^{m+1} \log q(1+|t|)\right) .
\end{aligned}
$$

We shall estimate the $2 k$-th moment of each term of this expression. We notice first that

$$
\begin{aligned}
\sum_{\chi}\left(\sum_{X \leq p<X^{3}} \frac{\Lambda_{X}(p) \chi(p)}{\sqrt{p} \cdot p^{i t}(\log p)^{m+1}}\right)^{2 k}=\sum_{\chi}\left|\sum_{X^{k} \leq n<X^{3 k}} \frac{\chi(n) b(n)}{\sqrt{n} n^{i t}}\right|^{2} \\
\quad \ll q \sum_{X^{k} \leq n<X^{3 k}} \frac{|b(n)|^{2}}{n} \ll q \frac{1}{(\log X)^{k m}}\left(\sum_{X \leq p<X^{3}} \frac{1}{p(\log p)^{m}}\right)^{k} \ll \frac{q}{(\log X)^{2 k m}},
\end{aligned}
$$

where we put

$$
b(n)=\sum_{n=p_{1} \cdots p_{j} \cdots p_{k}, X \leq p_{j}<X^{3}} \frac{\Lambda_{X}\left(p_{1}\right) \cdots \Lambda_{X}\left(p_{k}\right)}{\left(\log p_{1}\right)^{m+1} \cdots\left(\log p_{k}\right)^{m+1}}
$$

and use the estimate

$$
b(n) \ll \frac{1}{(\log X)^{k m}} .
$$

In a similar manner, we get

$$
\sum_{\chi}\left|\sum_{X \leq p^{2}<X^{3}} \frac{\Lambda_{X}\left(p^{2}\right) \chi\left(p^{2}\right)}{p \cdot p^{2 i t}\left(\log p^{2}\right)^{m+1}}\right|^{2 k} \ll \frac{q}{(\log X)^{2 k m}} .
$$

Thus we get

$$
\sum_{\chi}\left|\sum_{X \leq n<X^{3}} \frac{\Lambda_{X}(n) \chi(n)}{\sqrt{n} n^{i t}(\log n)^{m+1}}\right|^{2 k} \ll \frac{q}{(\log X)^{2 k m}} .
$$

Applying Lemma 17 on p. 331 of Selberg [5], we get

$$
\sum_{\chi \neq \chi_{0}}\left(\sigma_{\chi}-\frac{1}{2}\right)^{2 k(m+1)}(\log q(1+|t|))^{2 k} \ll \frac{q \log ^{2 k} q}{(\log X)^{2 k(m+1)}} .
$$

Combining these two types of the estimates, we can estimate the rest as follows. 


$$
\begin{aligned}
& \sum_{\chi \neq \chi_{0}}\left(\left(\sigma_{\chi}-\frac{1}{2}\right) X^{\sigma_{\chi}-\frac{1}{2}} \int_{\frac{1}{2}}^{\infty} X^{\frac{1}{2}-\sigma}\left|\sum_{n<X^{3}} \frac{\Lambda_{X}(n) \chi(n) \log (X n)}{n^{\sigma+i t}(\log n)^{m}}\right| d \sigma\right)^{2 k} \\
& \ll\left(\sum_{\chi \neq \chi_{0}}\left(\sigma_{\chi}-\frac{1}{2}\right)^{4 k} X^{4 k\left(\sigma_{\chi}-\frac{1}{2}\right)}\right)^{\frac{1}{2}} \\
& \cdot\left(\sum_{\chi}\left(\int_{\frac{1}{2}}^{\infty} X^{\frac{1}{2}-\sigma}\left|\sum_{n<X^{3}} \frac{\Lambda_{X}(n) \chi(n) \log (X n)}{n^{\sigma+i t}(\log n)^{m}}\right| d \sigma\right)^{4 k}\right)^{\frac{1}{2}} \\
& \ll\left(\sum_{\chi \neq \chi_{0}}\left(\sigma_{\chi}-\frac{1}{2}\right)^{4 k} X^{4 k\left(\sigma_{\chi}-\frac{1}{2}\right)}\right)^{\frac{1}{2}} \\
& \cdot\left(\frac{1}{(\log X)^{4 k-1}} \int_{\frac{1}{2}}^{\infty} X^{\frac{1}{2}-\sigma} \sum_{\chi}\left|\sum_{n<X^{3}} \frac{\Lambda_{X}(n) \chi(n) \log (X n)}{n^{\sigma+i t}(\log n)^{m}}\right|^{4 k} d \sigma\right)^{\frac{1}{2}} \\
& \ll \frac{q}{(\log X)^{2 k}} \begin{cases}(\log \log X)^{k} & \text { if } m=1 \\
1 & \text { if } m \geq 2,\end{cases}
\end{aligned}
$$

because we have

$$
\begin{aligned}
\sum_{\chi} \mid & \left.\sum_{p<X^{3}} \frac{\Lambda_{X}(p) \chi(p) \log (X p)}{p^{\sigma+i t}(\log p)^{m}}\right|^{4 k}=\sum_{\chi}\left|\sum_{n<X^{6 k}} \frac{\chi(n) b(n)}{n^{\sigma+i t}}\right|^{2} \\
& \ll q(\log X)^{4 k} \cdot\left(\sum_{p<X^{3}} \frac{\log p}{p \log ^{m} p}\right)^{2 k} \ll q(\log X)^{4 k} \begin{cases}(\log \log X)^{2 k} & \text { if } m=1 \\
1 & \text { if } m \geq 2\end{cases}
\end{aligned}
$$

with

$$
\begin{aligned}
b(n) & =\sum_{n=p_{1} \cdots p_{j} \cdots p_{2 k}, p_{j} \leq X^{3}} \frac{\Lambda_{X}\left(p_{1}\right) \cdots \Lambda_{X}\left(p_{2 k}\right) \log \left(X p_{1}\right) \cdots \log \left(X p_{2 k}\right)}{\left(\log p_{1} \cdots \log p_{2 k}\right)^{m}} \\
& \ll(\log X)^{2 k} .
\end{aligned}
$$

In the same manner, we have, for each $1 \leq v \leq m$,

$$
\begin{aligned}
\sum_{\chi \neq \chi_{0}} & \left(\left(\sigma_{\chi}-\frac{1}{2}\right)^{v} X^{\sigma_{\chi}-\frac{1}{2}} \int_{\frac{1}{2}}^{\infty} X^{\frac{1}{2}-\sigma}\left|\sum_{n<X^{3}} \frac{\Lambda_{X}(n) \chi(n) \log (X n)}{n^{\sigma+i t}(\log n)^{m+1-v}}\right| d \sigma\right)^{2 k} \\
\ll & \left(\sum_{\chi \neq \chi_{0}}\left(\sigma_{\chi}-\frac{1}{2}\right)^{4 k v} X^{4 k\left(\sigma_{\chi}-\frac{1}{2}\right)}\right)^{\frac{1}{2}} \\
& \cdot\left(\frac{1}{(\log X)^{4 k-1}} \int_{\frac{1}{2}}^{\infty} X^{\frac{1}{2}-\sigma} \sum_{\chi}\left|\sum_{n<X^{3}} \frac{\Lambda_{X}(n) \chi(n) \log (X n)}{n^{\sigma+i t}(\log n)^{m+1-v}}\right|^{4 k} d \sigma\right)^{\frac{1}{2}}
\end{aligned}
$$




$$
\ll \frac{q}{(\log X)^{2 k v}} \begin{cases}(\log \log X)^{k} & \text { if } v=m \\ 1 & \text { if } 1 \leq v \leq m-1 .\end{cases}
$$

Hence, we get

$$
\begin{gathered}
\sum_{\chi \neq \chi_{0}}\left(\sum_{\nu=1}^{m}\left(\sigma_{\chi}-\frac{1}{2}\right)^{v} X^{\sigma_{\chi}-\frac{1}{2}} \int_{\frac{1}{2}}^{\infty} X^{\frac{1}{2}-\sigma}\left|\sum_{n<X^{3}} \frac{\Lambda_{X}(n) \chi(n) \log (X n)}{n^{\sigma+i t}(\log n)^{m+1-\nu}}\right| d \sigma\right)^{2 k} \\
\ll \frac{q}{(\log X)^{2 k m}}(\log \log X)^{k}+\frac{q}{(\log X)^{2 k}} \\
\ll \frac{q}{(\log X)^{2 k}} \begin{cases}(\log \log X)^{k} & \text { if } m=1 \\
1 & \text { if } m \geq 2 .\end{cases}
\end{gathered}
$$

Finally, we have in the same manner

$$
\begin{aligned}
\sum_{\chi \neq \chi_{0}}( & \left.\left(\sigma_{\chi}-\frac{1}{2}\right)^{m+1} X^{\sigma_{\chi}-\frac{1}{2}} \int_{\frac{1}{2}}^{\infty} X^{\frac{1}{2}-\sigma}\left|\sum_{n<X^{3}} \frac{\Lambda_{X}(n) \chi(n) \log (X n)}{n^{\sigma+i t}}\right| d \sigma\right)^{2 k} \\
\ll & \left(\sum_{\chi \neq \chi_{0}}\left(\sigma_{\chi}-\frac{1}{2}\right)^{4 k(m+1)} X^{4 k\left(\sigma_{\chi}-\frac{1}{2}\right)}\right)^{\frac{1}{2}} \\
& \cdot\left(\frac{1}{(\log X)^{4 k-1}} \int_{\frac{1}{2}}^{\infty} X^{\frac{1}{2}-\sigma} \sum_{\chi}\left|\sum_{n<X^{3}} \frac{\Lambda_{X}(n) \chi(n) \log (X n)}{n^{\sigma+i t}}\right|^{4 k} d \sigma\right)^{\frac{1}{2}} \\
\ll & \frac{q}{(\log X)^{k(2 m+1)}} .
\end{aligned}
$$

Consequently, we get

$$
\begin{gathered}
\sum_{\chi \neq \chi_{0}}\left|I_{m}(t, \chi)-\frac{1}{\pi} \Im\left\{i^{m} \sum_{n<X} \frac{\Lambda(n) \chi(n)}{\sqrt{n} n^{i t}(\log n)^{m+1}}\right\}\right|^{2 k} \\
\ll \frac{q}{(\log q)^{2 k}} \begin{cases}(\log \log q)^{k} & \text { if } m=1 \\
1 & \text { if } m \geq 2 .\end{cases}
\end{gathered}
$$

We notice that

$$
\begin{aligned}
\sum_{\chi \neq \chi_{0}}( & \left.\frac{1}{\pi} \Im\left\{i^{m} \sum_{n<X} \frac{\Lambda(n) \chi(n)}{\sqrt{n} n^{i t}(\log n)^{m+1}}\right\}\right)^{2 k} \\
= & \frac{(-1)^{(m+1) k}}{(2 \pi)^{2 k}} \sum_{j=0}^{2 k}\left(\begin{array}{c}
2 k \\
j
\end{array}\right)(-1)^{j(m+1)} \sum_{\chi}\left(\sum_{n<X} \frac{\Lambda(n) \bar{\chi}(n)}{\sqrt{n} n^{-i t}(\log n)^{m+1}}\right)^{j} \\
& \cdot\left(\sum_{n<X} \frac{\Lambda(n) \chi(n)}{\sqrt{n} n^{i t}(\log n)^{m+1}}\right)^{2 k-j}-\left(\frac{1}{\pi} \Im\left\{i^{m} \sum_{n<X} \frac{\Lambda(n) \chi_{0}(n)}{\sqrt{n} n^{i t}(\log n)^{m+1}}\right\}\right)^{2 k}
\end{aligned}
$$




$$
\begin{aligned}
= & \frac{q-1}{(2 \pi)^{2 k}} \sum_{j=0}^{2 k}\left(\begin{array}{c}
2 k \\
j
\end{array}\right)(-1)^{(j+k)(m+1)} \sum_{\substack{n_{l}=2 \\
n_{1} \cdots n_{j}=n_{j+1} \cdots n_{2 k}}}^{\infty} \frac{\Lambda\left(n_{1}\right) \cdots \Lambda\left(n_{2 k}\right)}{n_{1} \cdots n_{j}\left(\log n_{1} \cdots \log n_{2 k}\right)^{m+1}} \\
& +O\left(\frac{q}{\log q}\right) .
\end{aligned}
$$

Hence, we get

$$
\begin{aligned}
\sum_{\chi \neq \chi_{0}}\left(I_{m}(t, \chi)\right)^{2 k}=\sum_{\chi \neq \chi_{0}}\left(I_{m}(t, \chi)-M+M\right)^{2 k} \\
=\sum_{\chi \neq \chi_{0}} \sum_{j=0}^{2 k}\left(\begin{array}{c}
2 k \\
j
\end{array}\right) M^{j}\left(I_{m}(t, \chi)-M\right)^{2 k-j} \\
=\sum_{\chi \neq \chi_{0}} M^{2 k}+\sum_{\chi \neq \chi_{0}} \sum_{j=0}^{2 k-1}\left(\begin{array}{c}
2 k \\
j
\end{array}\right) M^{j}\left(I_{m}(t, \chi)-M\right)^{2 k-j} \\
=\sum_{\chi \neq \chi_{0}} M^{2 k}+O\left(\sum_{j=0}^{2 k-1}\left(\begin{array}{c}
2 k \\
j
\end{array}\right)\left(\sum_{\chi \neq \chi_{0}} M^{2 k}\right)^{\frac{j}{2 k}}\right) \cdot\left(\sum_{\chi \neq \chi_{0}}\left(I_{m}(t, \chi)-M\right)^{2 k}\right)^{\frac{2 k-j}{2 k}} \\
=\frac{q}{(2 \pi)^{2 k}} \sum_{j=0}^{2 k}\left(\begin{array}{c}
2 k \\
j
\end{array}\right)(-1)^{(j+k)(m+1)} \\
\sum_{\substack{n_{1}=2 \\
n_{1} \cdots n_{j}=n_{j+1} \cdots n_{2 k}}}^{\infty} \frac{\Lambda\left(n_{1}\right) \cdots \Lambda\left(n_{2 k}\right)}{n_{1} \cdots n_{j}\left(\log n_{1} \cdots \log n_{2 k}\right)^{m+1}}+O\left(\frac{q}{\log q} R_{m}(q)\right),
\end{aligned}
$$

where we put

$$
M=\frac{1}{\pi} \Im\left\{i^{m} \sum_{n<X} \frac{\Lambda(n) \chi(n)}{\sqrt{n} n^{i t}(\log n)^{m+1}}\right\}
$$

and

$$
R_{m}(q)= \begin{cases}\sqrt{\log \log q} & \text { if } m=1 \\ 1 & \text { if } m \geq 2\end{cases}
$$

Thus we have obtained our theorem.

\section{References}

[1] A. Fujii, On the zeros of the Riemann zeta function, Comment. Math. Univ. St. Pauli 51 (2002), 1-17.

[2] A. Fujii, On the zeros of Dirichlet $L$-functions, to appear.

[ 3 ] A. Fujii, On the zeros of the Riemann zeta function II, Comment. Math. Univ. St. Pauli 52 (2003), 165190. 
[ 4 ] J. E. Littlewood, On the zeros of Riemann's zeta function, Proc. of Camb. Phil. Soc., 22 (1924), $295-318$.

[ 5 ] A. Selberg, Collected Papers, Springer Verlag, vol. 1, 1989.

[6] E. C. Titchmarsh, The theory of the Riemann zeta function (2nd ed. rev. by D. R. Heath-Brown), Oxford Univ. Press, 1951 (1988).

Department of Mathematics

Rikkyo University

Nishi-Ikebukuro, Toshimaku

Tokyo, 171-8501 Japan 